\title{
Multi-target simultaneous ISAR imaging based on compressed sensing
}

\author{
Gang Li', Qingkai Hou, Shiyou Xu and Zengping Chen
}

\begin{abstract}
Conventional range-Doppler (RD) inverse synthetic aperture radar (ISAR) imaging method utilizes coherent integration of consecutive pulses to achieve high cross-range resolution. It requires the radar to keep track of the target during coherent processing intervals (CPI). This restricts the radar's multi-target imaging ability, especially when the targets appear simultaneously in different observing scenes. To solve this problem, this paper proposes a multi-target ISAR imaging method for phased-array radar (PAR) based on compressed sensing (CS). This method explores and exploits the agility of PAR without changing its structure. Firstly, the transmitted pulses are allocated randomly to different targets, and the ISAR image of each target can be then reconstructed from limited echoes using CS algorithm. A pulse allocation scheme is proposed based on the analysis of the target's size and rotation velocity, which can guarantee that every target gets enough pulses for effective CS imaging. Self-adaptive mechanism is utilized to improve the robustness of the pulse allocation method. Simulation results are presented to demonstrate the validity and feasibility of the proposed approach.
\end{abstract}

\section{Introduction}

Inverse synthetic aperture radar (ISAR) can generate images of targets with high resolution in two dimensions. It usually transmits wideband waveform to obtain highrange resolution and utilizes coherent integration of multi-pulse to achieve high cross-range resolution [1]. This is the basic idea of range-Doppler (RD) imaging. It requires both appropriate rotation angle (usually $3^{\circ}-5^{\circ}$ for RD imaging) and consecutive pulses during coherent processing intervals $(\mathrm{CPI})[2]$.

To the best of our knowledge, most ISARs in practice can only generate images one target at a time. Since the $\mathrm{RD}$ imaging method requires consecutive echoes, the radar must track the target for a long period. The observing scene of radar is always a limited region defined by the radar's receiving range gate and the beam width. Therefore, the observing scene of ISAR must be focused on the target during the CPI. In some applications, the radar needs to observe several targets appearing in different observing scenes simultaneously, as shown in Fig. 1. Such scenes are quite common for military air defense radars or space surveillance radars. Modern PAR

\footnotetext{
* Correspondence: Ig19860521@163.com

College of Electronic Science and Engineering, National University of Defense Technology, Changsha, Hunan 410073, China
}

with active electronically scanning phased-array antenna can change the frequency and beam direction of signal between pulses readily [3]. Therefore, PARs are widely applied in multi-target radars, such as long-range air defense radars and missile-tracking radars.

Although PAR can achieve multi-target detection and tracking comfortably by allocating the pulses to each target, there are still some challenges in imaging them simultaneously. As is well known, the maximum pulse repetition frequency (PRF) of radar is limited by the observing distance to prevent range ambiguity. Meanwhile, the radar still needs to assign some pulses for target detection, tracking, and other functions [4]. Hence, the PAR's pulses may be inadequate for the coherent integration of each target. This paper aims to exploit the flexibility of PAR and to achieve better performance in multi-target imaging.

Recently, CS has drawn great attention in data acquisition and signal processing. It suggests that the signal can be sampled at sub-Nyquist rate and be reconstructed correctly if the signal is sparse or compressive in some basis or transform domain [5-9]. ISAR imaging based on CS is also an active research area since the targets often show sparse reflections and occupy only limited pixels in the imaging results $[10,11]$. It has been proposed in [12] that ISAR image can be reconstructed
照 Springer

(c) $2016 \mathrm{Li}$ et al. Open Access This article is distributed under the terms of the Creative Commons Attribution 4.0 International License (http://creativecommons.org/licenses/by/4.0/), which permits unrestricted use, distribution, and reproduction in any medium, provided you give appropriate credit to the original author(s) and the source, provide a link to the Creative Commons license, and indicate if changes were made. 


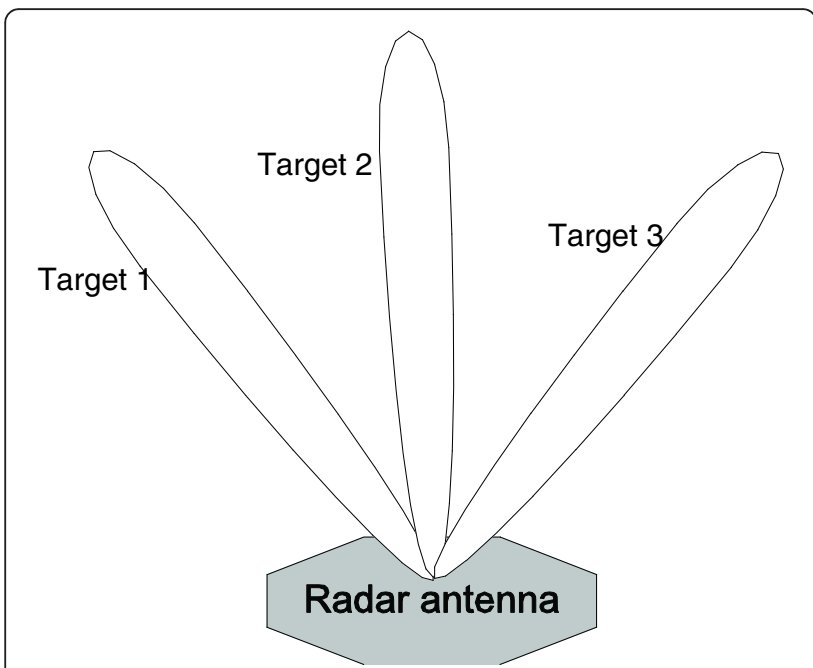

Fig. 1 Scenario of multi-target for PAR ISAR radar

using much fewer pulses than RD algorithm with random pulse repetition interval (PRI). At present, most of the research on CS imaging is aimed at a single target and has achieved some research results [7-10, 12]. Considering the requirement of radar multi-target observation and imaging, this paper tries to use the CS imaging method to improve the ability of simultaneous multitarget radar imaging.

Inspired by CS and the flexibility of PAR, we allocate the pulses randomly to multiple targets appearing in different observing scenes. The radar illuminates one target at one pulse and changes target from pulse to pulse. The observing sequence is arranged randomly to realize non-uniform sampling in the cross-range dimensions of each target. Such random sampling can insure that the measurement matrix satisfies the restricted isometry property (RIP), which is the sufficient criterion for effective reconstruction in CS [13, 14]. Another major contribution of this paper is that we propose a pulse allocating strategy based on detailed analysis of CS ISAR imaging. Besides random sampling, the sizes and rotation velocities of targets are also determining factors for pulse allocation. This allocation of pulse guarantees that every target obtains enough measurements for effective CS reconstruction. The key innovation of this method is fully utilizing the limited resource of radar pulses to implement multi-target imaging, based on the image sparsity and CS algorithms. After random observation, the measurement matrices for the targets are different from each other; thus, the images are reconstructed separately using CS algorithms. Experiment results are provided to demonstrate the validity of this observing strategy and imaging method.
This paper is organized as follows. In Section 2, the mathematic model of ISAR imaging is built and the CS imaging method is presented. In Section 3, the multitarget observing scheme and reconstructing method of ISAR image are presented based on CS. The experiment results and analysis are provided in Section 4, and conclusions are given in Section 5.

\section{CS ISAR imaging using limited pulses}

\subsection{Model of range-Doppler ISAR imaging}

Assume that the translational motion of the target has been compensated using conventional methods [15-17]. The target can be treated as a platform which rotates around the center $O$, as shown in Fig. 2 . Suppose the transmitted linear frequency modulation (LFM) signal is

$$
S_{T}(t)=\operatorname{rect}\left(\frac{t}{T_{\mathrm{P}}}\right) \exp \left(j 2 \pi\left(f_{c} t+\frac{1}{2} \gamma t^{2}\right)\right)
$$

where $\operatorname{rect}(u)=\left\{\begin{array}{ll}1, & |u| \leq 1 / 2 \\ 0, & |u|>1 / 2\end{array}, f_{c}\right.$ represents the center frequency, $T_{\mathrm{p}}$ is the pulse width, $\gamma$ is he frequency modulation rate, and $t$ is the fast time. The echo reflected by the scattering point locating at $P(x, y)$ after matched filtering pulse compression can be written as

$$
s_{\mathrm{R}}\left(t, t_{\mathrm{s}}\right)=\beta \operatorname{sinc}\left(T_{\mathrm{p}} \gamma\left(t-\frac{2 R\left(t_{\mathrm{s}}\right)}{c}\right)\right) \exp \left(-j 4 \pi \frac{R\left(t_{\mathrm{s}}\right)}{\lambda}\right)
$$

where $\beta$ is the backward scattering intensity of $P(x, y), \lambda$ is the wavelength of signal, $t$ is the fast time, and $t_{\mathrm{s}}$ is the slow time. $R\left(t_{\mathrm{s}}\right)$ is the instantaneous distance from $P(x, y)$ to radar and at $t_{\mathrm{s}}$

$$
R\left(t_{\mathrm{s}}\right)=R_{0}+y \cos \theta\left(t_{\mathrm{s}}\right)+x \sin \theta\left(t_{\mathrm{s}}\right)
$$

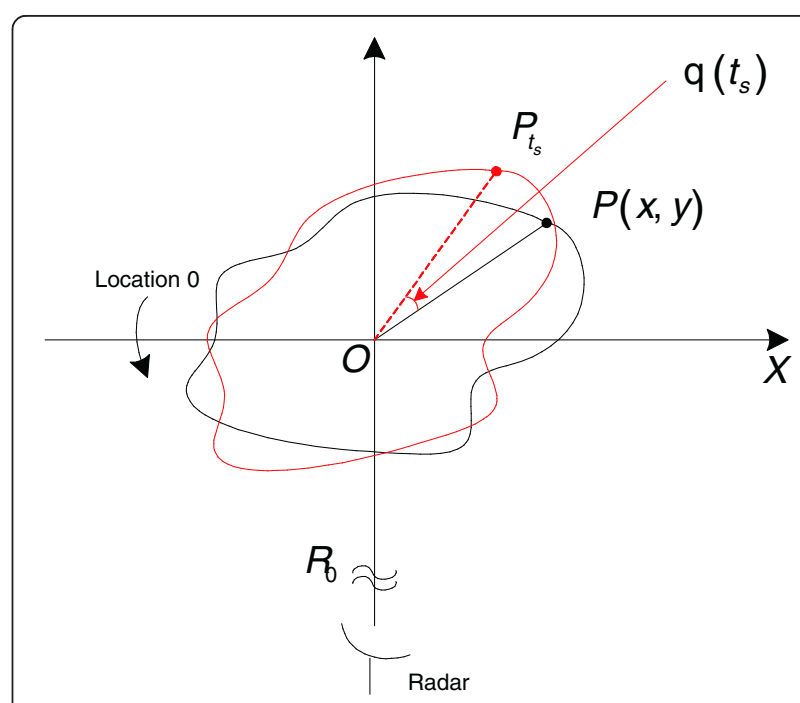

Fig. 2 Geometry of the scattering point $p$ 
where $\theta\left(t_{\mathrm{s}}\right)$ is the instantaneous rotation angle of the $m$ th pulse. Since the rotation angle is usually very small in ISAR, the $R\left(t_{\mathrm{s}}\right)$ can be approximated as

$$
R\left(t_{\mathrm{s}}\right) \approx R_{0}+y+x \theta\left(t_{\mathrm{s}}\right)
$$

In Eq. (4), $\theta\left(t_{\mathrm{s}}\right)$ can be approximated by Taylor expansion as

$$
\theta\left(t_{\mathrm{s}}\right) \approx \omega t_{\mathrm{s}}+0.5 \alpha t_{\mathrm{s}}^{2}+\sigma\left(t_{\mathrm{s}}^{2}\right)
$$

where $\omega$ and $\alpha$ are rotation velocity and acceleration, respectively. Therefore, the echo can be approximated as

$$
\begin{aligned}
s_{\mathrm{R}}\left(t, t_{\mathrm{s}}\right) \approx \beta & \sin c\left(T_{\mathrm{P}} \gamma\left(t-\frac{2\left(R_{\mathrm{o}}+y\right)}{c}\right)\right) \\
& \quad \exp \left(-j 4 \pi\left(\frac{R_{\mathrm{o}}+y+x\left(\omega t_{\mathrm{s}}+0.5 \alpha t_{\mathrm{s}}^{2}\right)}{\lambda}\right)\right)
\end{aligned}
$$

Suppose the total number of range cells is $M$ and there are $K$ scatters in the $m$ th range cell. The rotational motion is assumed to be stationary. After neglecting the constant term and high-order term, the signal of the $m$ th range cell can be denoted as

$$
\begin{aligned}
& s_{m}\left(t_{\mathrm{s}}\right) \approx \sum_{k=1}^{K} \beta \operatorname{sinc}\left(T_{\mathrm{P}} \gamma\left(t-\frac{2\left(R_{0}+y\right)}{c}\right)\right) \\
& \exp \left(-j 4 \pi\left(\frac{R_{0}+y+0.5 \alpha t_{\mathrm{s}}^{2}}{\lambda}\right)\right) \\
& \exp \left(-j 2 \pi\left(\frac{2 x_{k} \omega}{\lambda}\right) t_{\mathrm{s}}\right) \\
& \approx \sum_{k=1}^{K} \beta \operatorname{sinc}\left(T_{\mathrm{P}} \gamma\left(t-\frac{2\left(R_{0}+y\right)}{c}\right)\right) \\
& \exp \left(-j 4 \pi\left(\frac{R_{0}+y}{\lambda}\right)\right) \exp \left(-j 2 \pi\left(\frac{2 x_{k} \omega}{\lambda}\right)\right. \\
& =\sum_{k=1}^{K} \beta_{k} \exp \left(-j 2 \pi\left(\frac{2 x_{k} \omega}{\lambda}\right) t_{\mathrm{s}}\right)
\end{aligned}
$$

where $\quad \beta_{k}=\beta \operatorname{sinc}\left(T_{\mathrm{P}} \gamma\left(t-\frac{2\left(R_{0}+y\right)}{c}\right)\right) \exp \left(-j 4 \pi\left(\frac{R_{0}+y}{\lambda}\right)\right)$, which denotes the $k$ th scattering point's intensity. In RD imaging, the cross-range compression is achieved by applying a Fourier transform in the cross-range dimension. Let $f_{k}=\frac{2 x_{k} \omega}{\lambda}$ denote the Doppler frequency of the $k$ th scatter, and we can achieve the compression result of the $m$ th range cell as

$$
s_{m}\left(f_{d}\right)=\sum_{k=1}^{K} \beta_{k} \delta\left(f_{d}-f_{k}\right)
$$

Considering the sampling window in the cross-range domain, the $\delta(\bullet)$ function will be substituted by $\sin c(\bullet)$.
If we build a dictionary $\Psi$ and the $i$ th column of $\Psi$ is $\psi_{i}=\exp \left[-j 2 \pi f_{d}(i) t_{s}\right]$, Eq. (7) can be rewritten in the matrix form as

$$
s_{m}=\Psi \beta_{m}+n
$$

where $s_{m}$ is the vector form of $s_{m}\left(t_{s}\right), \beta_{m}$ is the vector which denotes the scatters distribution in the $m$ th range cell, and $n$ is the measuring noise. The whole image of the target is obtained after cross-range compression of all $M$ range cells.

\subsection{CS ISAR imaging using random pulses}

In most ISAR cases, there are only a few strong scattering points in one range cell, and the number of scattering centers is much smaller than the number of pulses. Therefore, the vector $\beta$ is sparse, and the echoes of target are compressive in the cross-range dimension. CS ISAR imaging method has been proposed in [12] to reconstruct the image using much fewer pulses than RD algorithm with random PRI. In this paper, further study about CS ISAR imaging is carried on and some conditions are given for effective CS imaging.

First, to achieve effective reconstruction in CS, the number of measurements must exceed the theoretical minimal number [6].

$$
M>O(K \cdot \log N)
$$

where $K$ is the sparse level and $N$ is the length of the measured sparse signal. In the context of CS ISAR imaging, the sparse level $K$ is the number of cross-range bins which are occupied by target in image, and Eq. (10) determines the minimal number of transmitted pulses for the target.

Second, the cross-range resolution in conventional RD

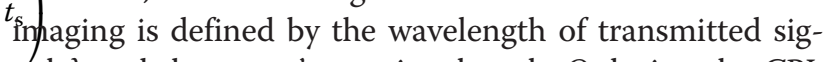
nal $\lambda$ and the target's rotational angle $\Theta$ during the CPI, which is denoted as

$$
\Delta_{c}=\frac{\lambda}{2 \Theta}
$$

The rotation angle $\Theta$ is usually $3^{\circ}-5^{\circ}$ because the smaller angle is inadequate for high resolution and the migration through resolution cell (MTRC) will occur if $\Theta$ is larger [18]. Moreover, the sampling rate in the cross-range dimension, e.g., the PRF of radar, must be at least twice the Doppler bandwidth of the target, which is proportional to the target's rotation velocity. Hence, targets with different rotation velocities need different PRFs for equivalent cross-range resolution. Faster rotation means that the CPI for one image is shorter and a higher PRF is required. The reference [19] suggests that the expected resolution of CS reconstructed image is restricted by the incoherency of the sensing matrix. In CS ISAR 
imaging, since the random pulses are the subset of Nyquist-sampled pulses in RD imaging, Eq. (11) still determines the cross-range resolution, and enough rotation angle is also required for high resolution.

According to the above analysis, we give the following two conditions that the random measurements must satisfy to achieve effective CS ISAR imaging.

2.2.0.1 Condition 1. The number of random pulses $M$ is more than the minimal measurements needed by CS, as (10).

2.2.0.2 Condition 2. The rotation angle of the target during CPI is approximately $3^{\circ}-5^{\circ}$, which is the same as RD method. This guarantees the reconstructed image own the same cross-range resolution as RD imaging result.

\section{CS multi-target simultaneous imaging}

\subsection{Basic idea of multi-target observation}

Based on the CS ISAR imaging, a multi-target imaging method is proposed for PAR in this section. As introduced in Section 1, there may be multiple targets in different observing scenes, but the radar can only observe one target at one pulse. Instead of illuminating one target during the whole CPI, the pulses can be allocated to all the targets and the observing sequence is arranged randomly. Therefore, the PRI for each target is nonuniform and the measurements of every target are random sampling, which coincides with the CS imaging method condition for single target in [12].

Suppose there are four different targets, and Fig. 3 shows the sequence of transmitted pulses, which are allocated randomly. As a simple example, the pulses are allocated to four targets with equal proportion in Fig. 3, e.g., the probability of being illuminated for each target is equal. However, such an equal proportion is apparently too simple for the practical applications. The emphasis of this section is to determine the allocating proportions of pulses for different targets.

In the train of transmitted pulses, the illuminated target of each pulse can be treated as an independent and identically distributed (I.I.D.) random variable $A$. $A \in\{0,1,2 \cdots L\}$, where $L$ is the number of targets. $A_{m}=i(0<i \leq L)$ means the $m$ th pulse illuminates the $i$ th target, while $A_{m}=0$ means no target is observed, e.g., this pulse is not used in any target's imaging. The probability distribution of $A$ determines the allocation proportions of pulses for different targets.

To obtain effective CS measurements for each target, random allocation of pulses must ensure that the measurements of every target satisfy the two conditions presented in Section 2. Based on this, we propose an algorithm to determine the probability distribution of $A$.

For condition 1, the key problem is to determine the sparse level of target, namely the size of target in cross-range dimension. For coarse estimation, the target's size can be estimated from the radar crosssection (RCS) [20] and high-resolution range profiles (HRRPs) of the targets. Although these methods cannot provide accurate estimation of the cross-range size of target, they can help to determine the sparse level of target roughly. Together with the expected resolution of ISAR image $\Delta_{c}$, the sparse level of target $k_{i}$ can be estimated by

$$
K_{i}=D_{i} / \Delta_{c}
$$

where $D_{i}$ is the estimated size of target. Therefore, the number of pulses $M_{i}$ needed for each target can be calculated in Eq. (10).

As for condition 2, the CPI of a target depends on its rotation velocity $\omega$ with respect to the radar line of sight. For space objects, the rotation velocity $\omega$ can be estimated from the orbit information. While for other non-cooperate targets, the $\omega$ can be estimated from Doppler analysis of target's echoes. Reference [21] suggests that the received signal of range bin can be approximated as cubic phase signals, and the ratio of the third-order phase coefficient to the first-order phase coefficient is just the square of the rotation velocity. We use this algorithm to estimate the rotation velocity $\omega_{i}$ of each target, and the CPI $\tau_{i}$ of target can be obtained by

$$
\tau_{i}=\frac{\bar{\Theta}}{\omega_{i}}
$$

where $\bar{\Theta}$ is the typical rotation angle for ISAR imaging and is set to be $\bar{\Theta}=4^{\circ}$.

Based on those estimations, the least number of pulses and CPIs of each target are determined. We use $\bar{\rho}_{i}$ to denote the equivalent average PRF for the $i$ th target, and $\bar{\rho}_{i}$ is given as

$$
\bar{\rho}_{i}=\frac{M_{i}}{\tau_{i}}
$$

It proposes that the allocating probability for different target is proportional to the equivalent average $\operatorname{PRF} \bar{\rho}_{i}$ of each target. The probability distribution of $A_{m}$ follows

$$
P\left(A_{m}=i\right)=\frac{\bar{\rho}_{i}}{f_{\mathrm{PRF}}}
$$

where $f_{\mathrm{PRF}}$ is the actual PRF with Nyquist sampling rate in the conventional RD imaging. Besides, the maximum number of targets that the radar can observe simultaneously can be derived as 


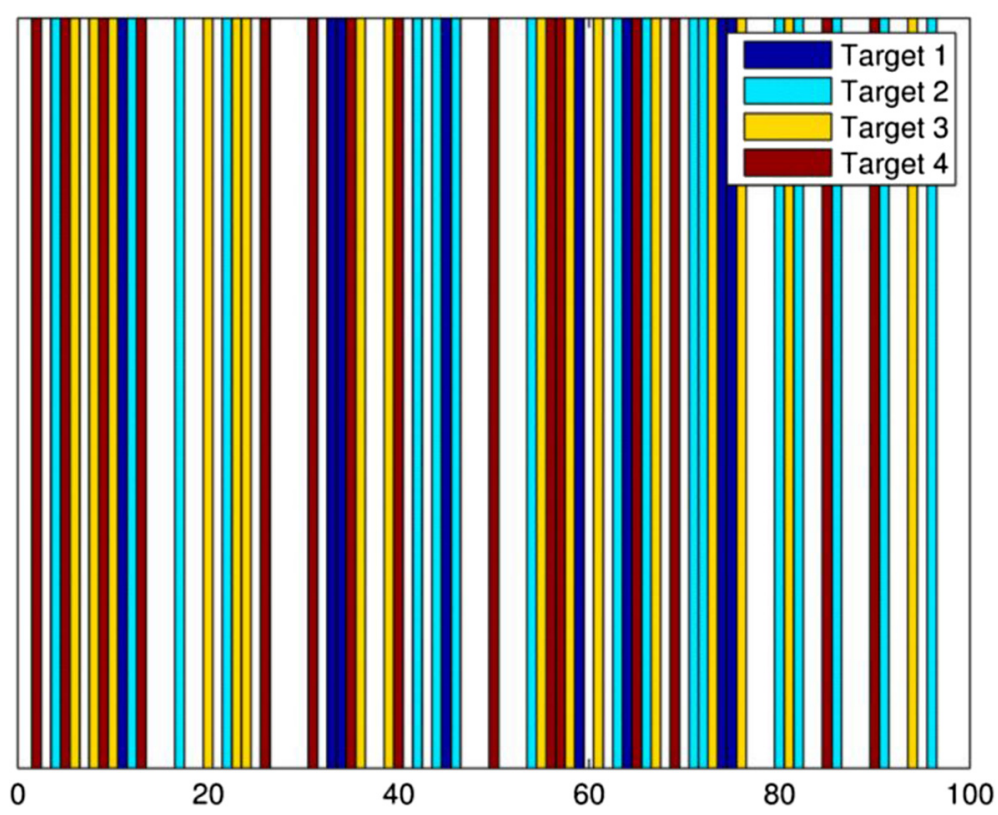

Fig. 3 An example of random pulse allocation for four targets

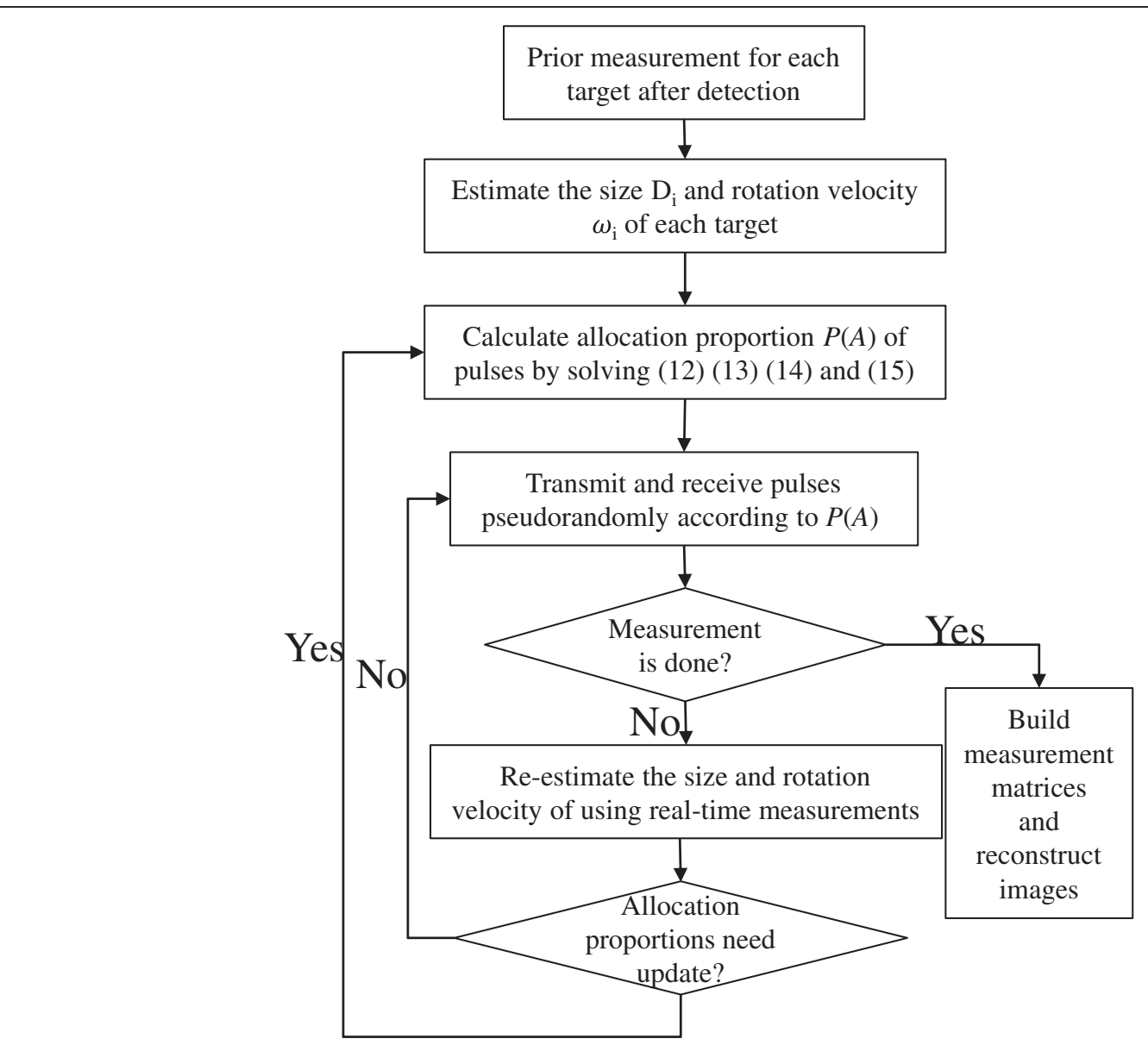

Fig. 4 Flow chart of compressive measurement for multiple targets 


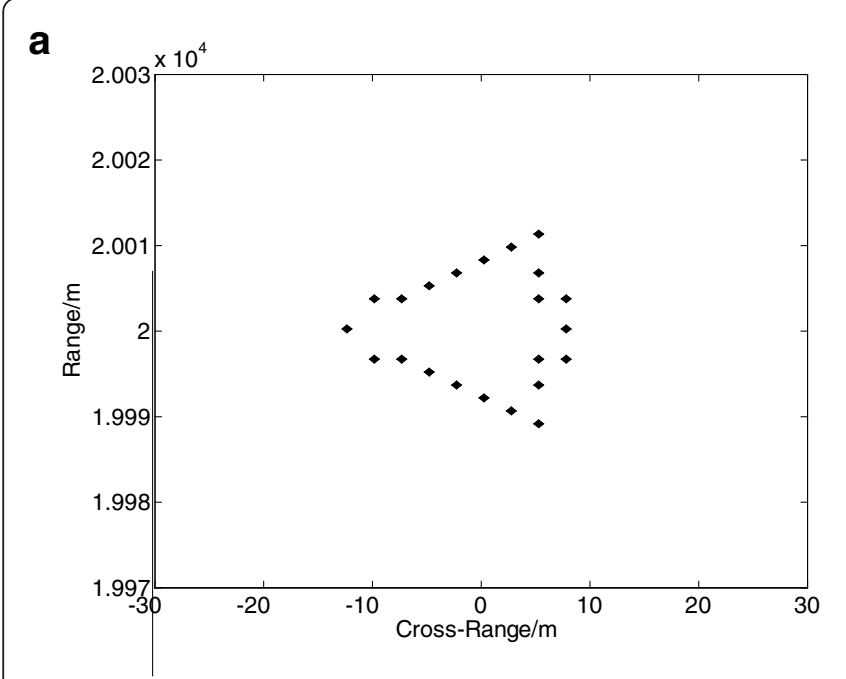

b

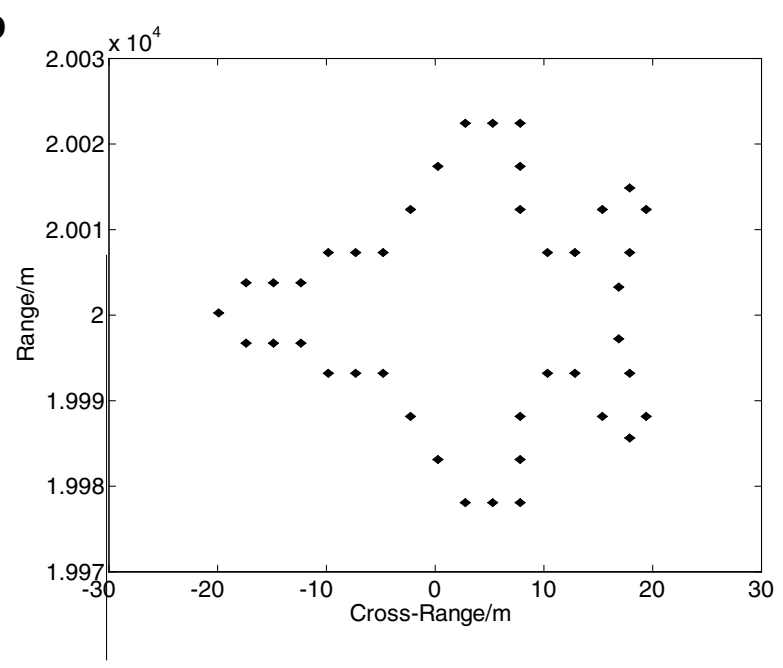

C

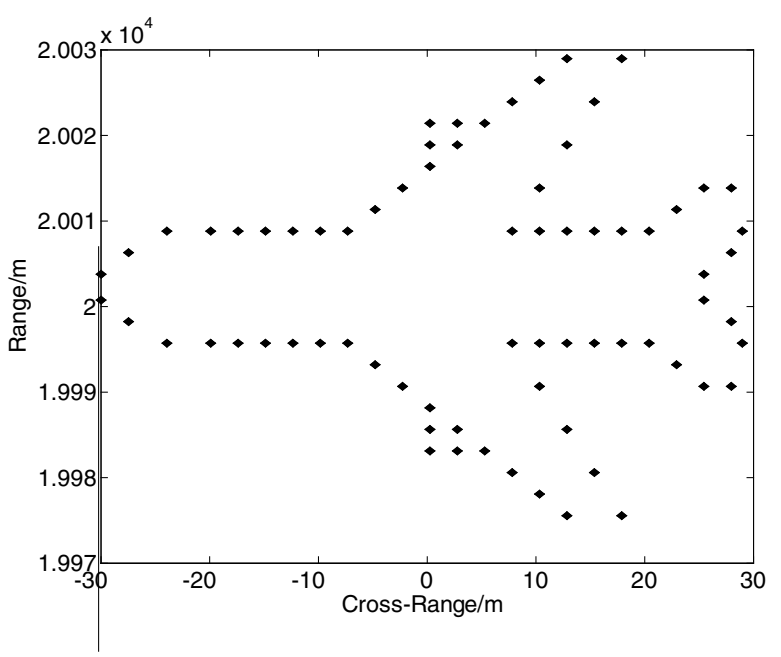

d

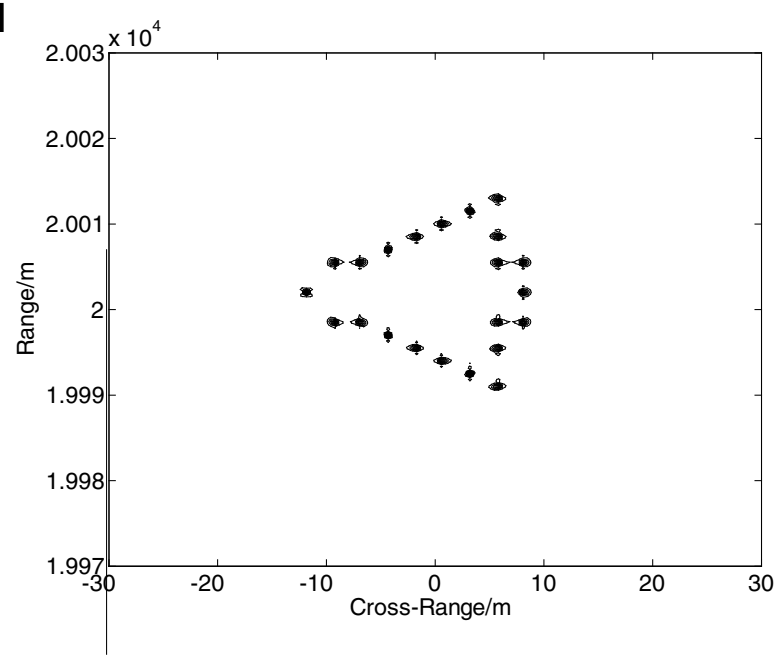

$\mathbf{e}$

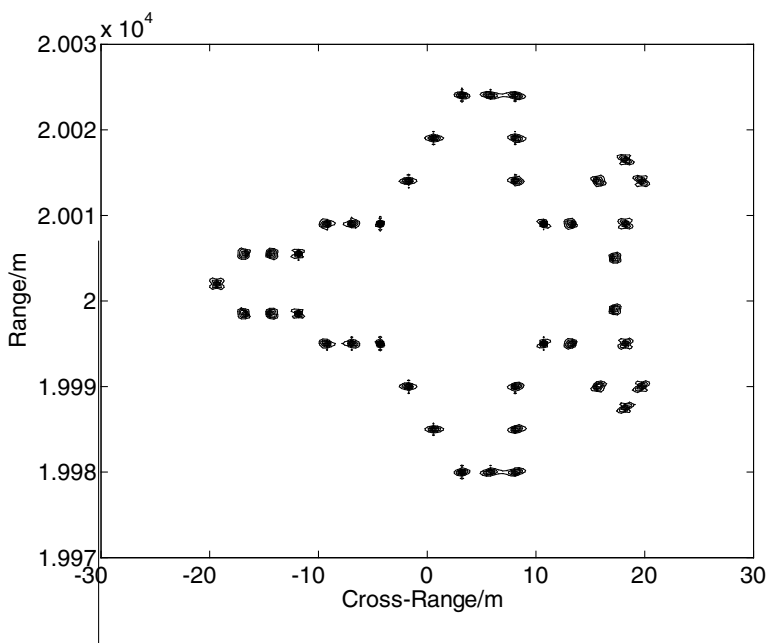

f

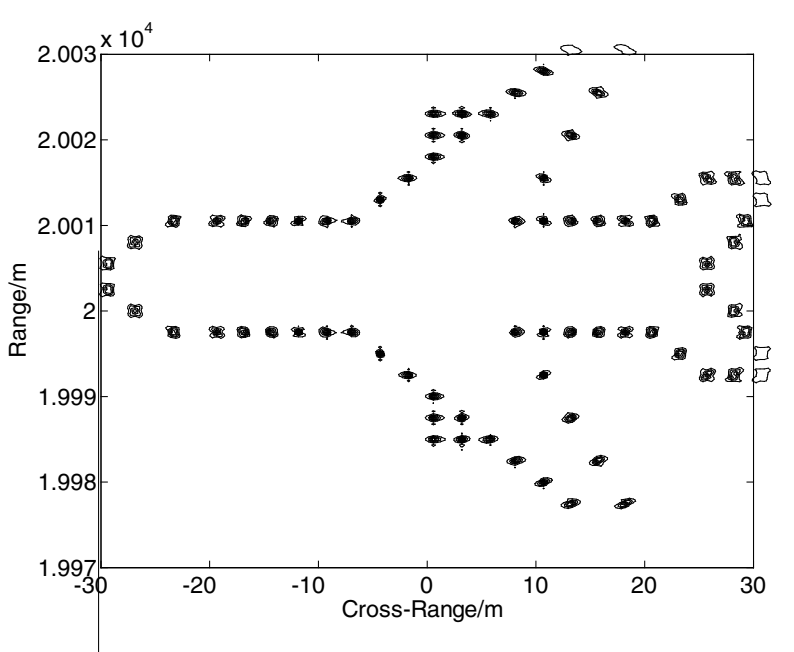

Fig. 5 Reflectivity distribution and conventional RD imaging results of three simulated targets. (a)-(c) are the point scattering models of the three targets; (d)-(f) are the conventional RD imaging results of three simulated targets 


$$
\sum_{i=1}^{L} \bar{\rho}_{i} \leq f_{\mathrm{PRF}}
$$

If targets number $L$ exceeds this limitation, the pulse allocation cannot guarantee that enough measurements are acquired for each target.

For the convenience of radar management, the estimation of sparse level and rotation velocity can be obtained as prior information for multi-target CS imaging. Specifically, this can be done after the radar detection and tracking process; then, the wideband pulses for imaging are transmitted pseudo-randomly according to the estimated probability density $P\left(A_{m}\right)$.

\subsection{Adaptive pulse allocating method}

The estimations of rotation velocity and target size are very important for the multi-target CS imaging method. However, the estimation results in practice are usually inaccurate due to the influence of noise and clutters. Besides, the prior measurements before imaging may be insufficient or incorrect to determine the appropriate pulse allocating proportions. Considering this, an improved adaptive observing strategy for multiple targets is introduced in this section. With the development of digital processing, the modern radar systems can own more powerful real-time processing ability. During CS imaging, some real-time analysis can be carried out to estimate the velocities and sizes of the targets. This allows us to update the allocating proportions of pulses during observation.

In three situations, we need to re-calculate the allocating proportions for different targets. (1) Some targets disappear from the radar's view and do not need imaging anymore; then, it is eliminated from the targets list. (2) A new target is detected and is added into the imaged targets list. (3) The estimations of targets' velocities and sizes are considerably different from the current ones.

The self-adapting allocation proportions can improve the robustness of the multi-target observing method. For convenience of understanding, a flow chart is presented to interpret the work mode of radar in the adaptive CS imaging method, as shown in Fig. 4.

The sequence of pulses must be memorized in the radar system because it is essential to build the measurement matrices of each target, which will be discussed in Section 3.3.

Limited by the real-time capability of the radar system and the complexity of CS reconstructing algorithms, it is difficult to realize real-time image reconstruction using limited pulses. Once it is solved with faster algorithms, the iterative method can be employed in the flow of multi-target observation. Instead of estimating the sizes and velocities of targets based on the RCS and HRRPs, the quality of real-time imaging results can be employed to evaluate whether the pulse allocation proportion is effective. In the following observation, more pulses will be allocated to those targets with blurred images. The most reasonable proportions will be established after several iterations. This is the unsolved issue that we are investigating in the future work.

\subsection{Reconstruction images of multiple targets}

In ISAR imaging, the non-cooperative motion of target must be compensated to make the echoes consistent with the model of rotated platform in Fig. 2. References [22] and [23] suggest the method that minimizes entropy of average range profile, and the eigenvector method can be utilized to solve the range alignment and phase correction of random pulses. Reference [24] proposed a method to solve the problem of motion compensation when pulses are inconsecutive. Combining Eq. (9), the echoes after compensation can be denoted as

$$
y_{m}=\Phi \Psi \beta_{m}+n
$$

where matrix $\Phi$ is the random measurement matrix, and vectors $y_{m}$ and $n$ are the measurements of $m$ th range cell and the measured noise, respectively. The crossrange image $\beta_{m}$ of $m$ th range cell can be reconstructed by solving

$$
\min \left\|\hat{\beta}_{m}\right\|_{1} \quad \text { s.t. }\left\|y_{m}-\Phi \Psi \beta\right\|_{2} \leq \varepsilon
$$

where $\hat{\beta}_{m}$ is the reconstruction of vector $\beta$ and $\varepsilon$ is the noise level. It is the typical optimization problem in CS and there are some algorithms to solve it, such as basis pursuit (BP), orthogonal matching pursuit (OMP), Bayesian algorithms [25], and smoothed $\ell_{0}$ algorithm (SL0). The SL0 [26] has a good tradeoff between accuracy and complexity [27]. Different from most greedy algorithms and BP algorithms, the sparsity level of an original signal is not necessary for SL0 method. Therefore, it is quite suitable for CS radar imaging, since the number of scattering centers is unknown until the image is obtained.

The first step of reconstructing multi-target images is to build the measurement matrices for each target. In conventional Nyquist sampling, the measurement matrix is the identity matrix $I_{i}$, and the size $N_{i}$ of matrix $I_{i}$ is

Table 1 Parameter setting of three targets in simulation

\begin{tabular}{llll}
\hline Target & Range $(\mathrm{km})$ & Rotation velocity 1 (\%) & Rotation velocity 2 (\%) \\
\hline Target 1 & 100 & 2.0 & 1.0 \\
Target 2 & 120 & 2.0 & 2.5 \\
Target 3 & 90 & 2.0 & 5.0 \\
\hline
\end{tabular}



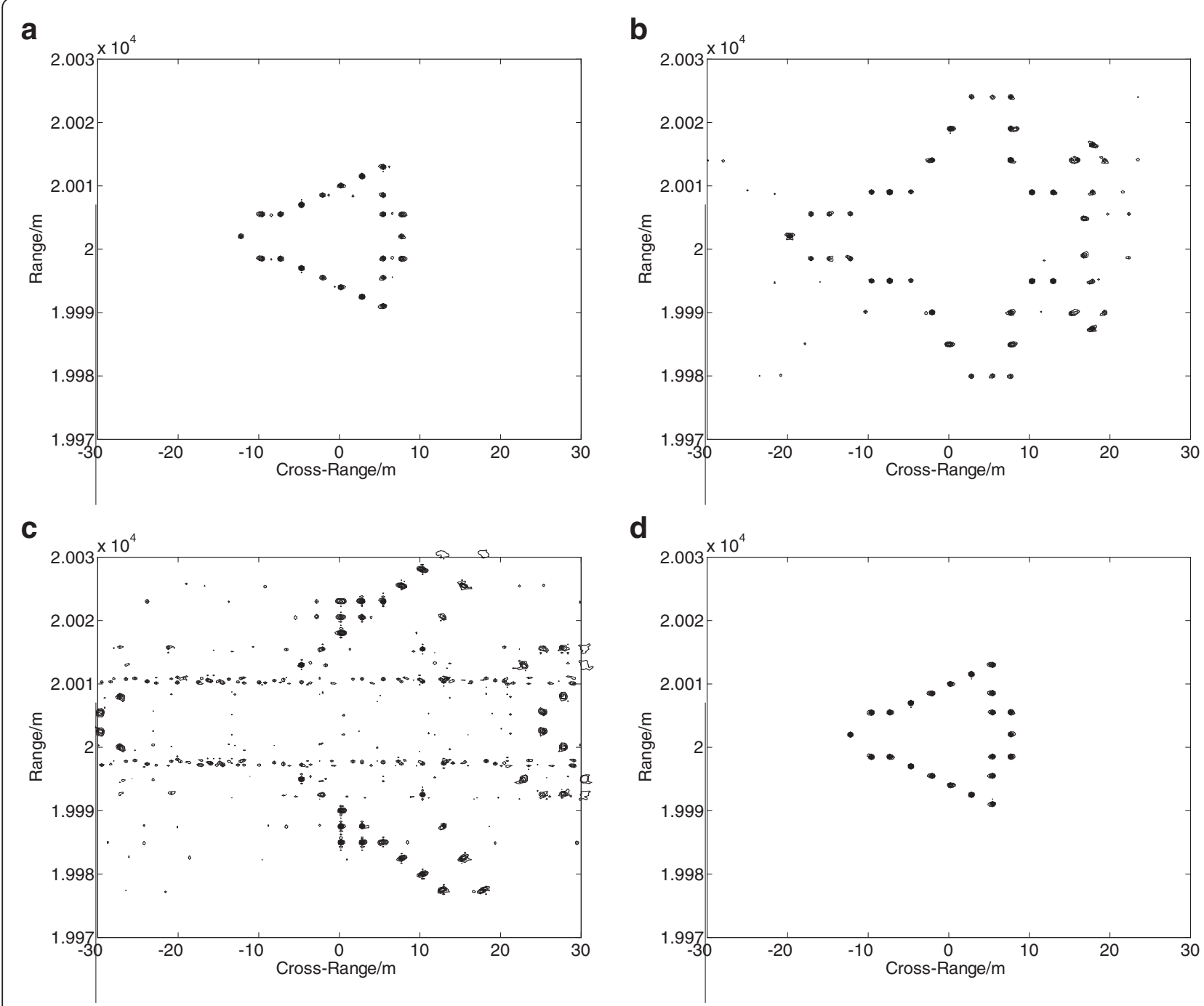

d

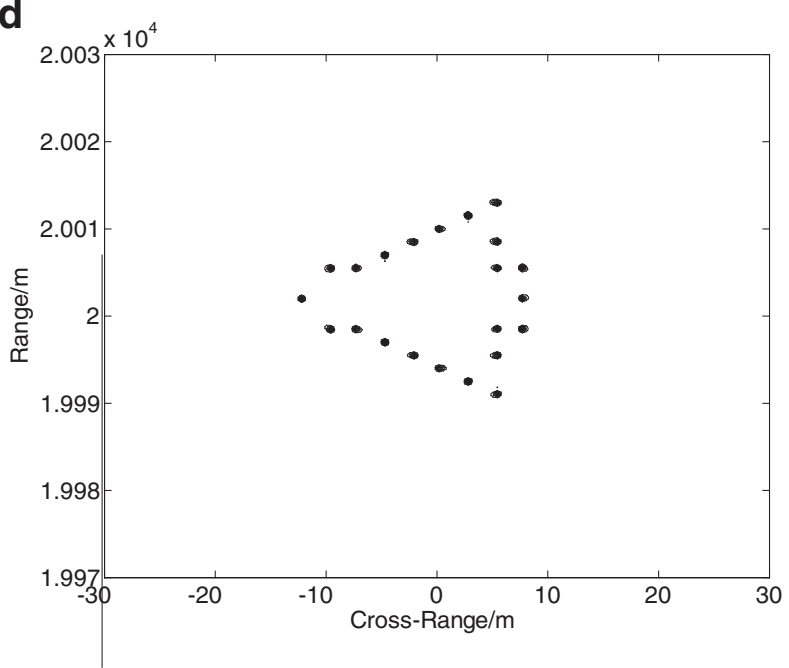

e

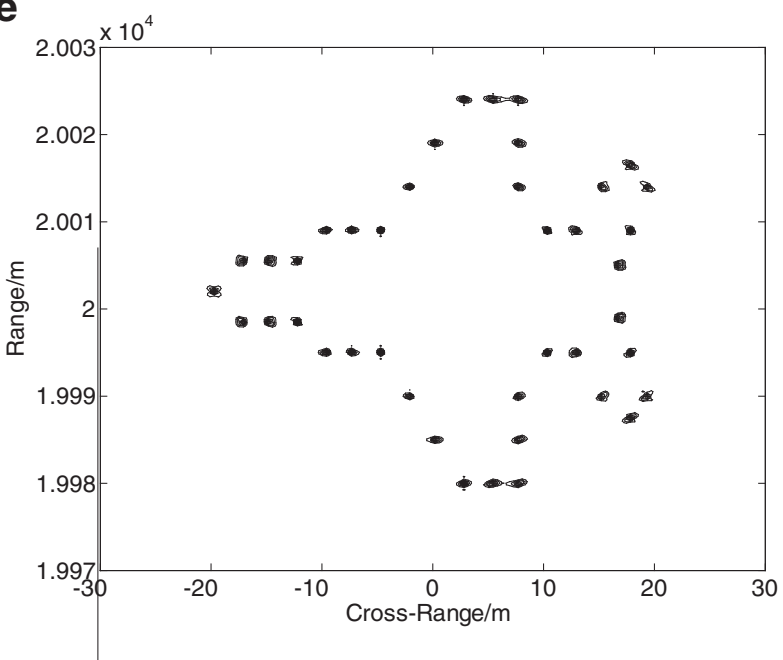

f

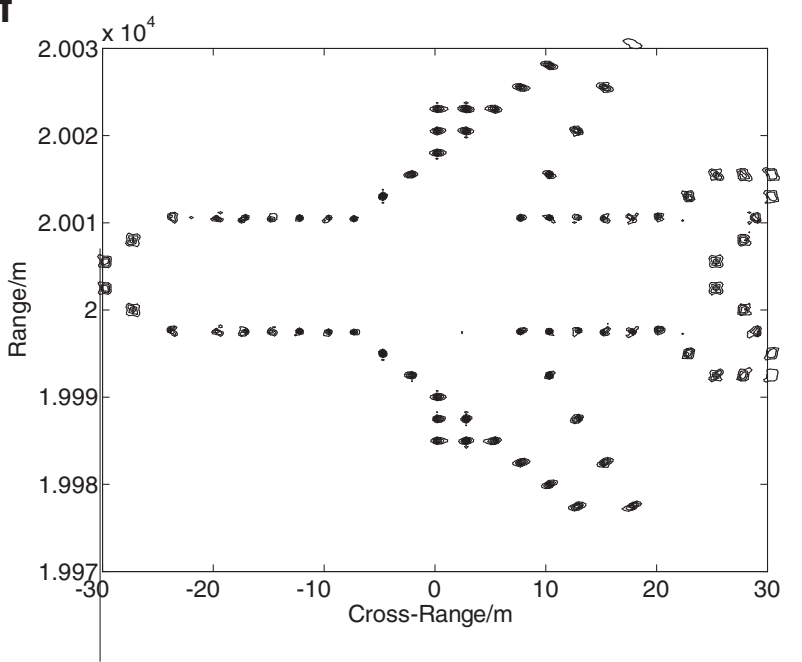

Fig. 6 The pulse allocating proportion affected by the size of target. a-c Reconstructed images when pulses are allocated randomly to three targets with the same probability. $\mathbf{d}-\mathbf{f}$ Reconstructed images when the allocating proportions are decided by the size of targets 

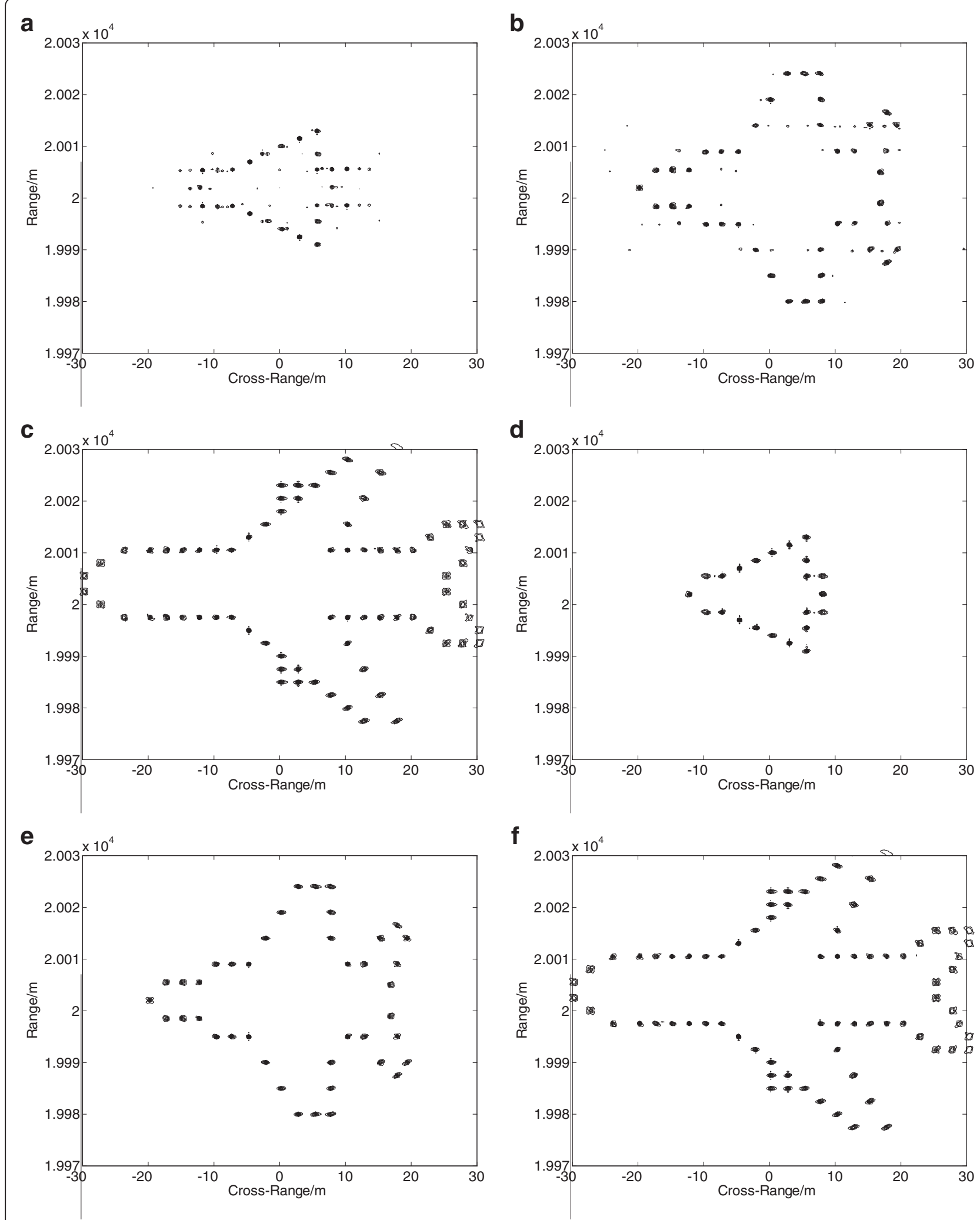

Fig. 7 The pulse allocating proportion affected by the rotation velocity of target. a-c Reconstructed images when the pulses are allocated without consideration of the different rotation velocities of targets. $\mathbf{d}-\mathbf{f}$ CS reconstructed images when the pulses allocation proportion is calculated according to both the sizes and the different rotation velocities of targets 
the number of pulses transmitted during corresponding CPI $\tau_{i}$, and

$$
N_{i}=f_{\mathrm{PRF}} \times \tau_{i}
$$

In CS multi-target imaging, the pulses are randomly allocated in CPI for each target. Hence, the measurement matrix $\Phi_{i}$ of the $i$ th target's echo in Eq. (17) can be obtained by selecting corresponding rows from $I_{i}$ according to the random sequence of pulses. The selected indices of rows are determined by the indices of the pulses that illuminate the $i$ th target.

Then, the images can be reconstructed by solving Eq. (18) for each target separately. The reconstructing performance and analysis will be given in Section 4.

\section{Simulation results}

To verify the validity of the proposed observing strategy and imaging method, some simulations are performed in this section. Suppose the parameters of the transmitted LFM signal are as follows. The bandwidth is $B=300 \mathrm{MHz}$, the carrier frequency is $f_{c}=10 \mathrm{GHz}$, the pulse width is $\tau_{c}=50 \mu \mathrm{s}$, and the PRF of radar is $f_{\mathrm{PRF}}=1000 \mathrm{~Hz}$.

In the simulation, three aeroplanes with different sizes are selected as the targets, which locate in different observing scenes. The point scattering models of the three targets are given in Fig. 5. The parameters of the three targets are presented in Table 1. As shown in Fig. 5, the sizes of three targets are different, and the sparse level $K_{i}$ of target is determined by the number of scattering points in the cross-range dimension. The simulations are carried out in two steps.

In the first experiment, the simulation is carried out to testify the effectiveness of target sizes on the allocation proportions of pulses. The rotation velocities of three targets are set to be the same, as shown in the third column of Table 1. If we allocate the radar pulses equally, without considering the difference of target sizes, the reconstructed images of three targets are shown in Fig. $6 \mathrm{a}-\mathrm{c}$. As we can see, target 3 has the biggest size, and the corresponding reconstructed image is blurred seriously. However, the images of the other two targets are well focused. Then, we use Eq. (15) to calculate the allocating proportions for three targets, and the proportion is about 2:3:4. As shown in Fig. $6 \mathrm{~d}-\mathrm{f}$, all targets are allocated with enough pulses, and thus, the images are all well focused.

In the second experiment, the difference of rotation velocities is considered and the rotation velocities of the three targets are set as the fourth column of Table 1 . The CPIs of three targets can be calculated to be about $\tau_{1}=5.0 \mathrm{~s}, \tau_{2}=2 \mathrm{~s}$, and $\tau_{3}=1 \mathrm{~s}$, respectively. First, the allocating probability is calculated by Eq. (15) without considering the difference of rotation velocities. As shown in Fig. $7 \mathrm{a}-\mathrm{c}$, the reconstructed images of targets 1 and 2 are blurred. The reason is that the two targets rotate much faster than target 3 and the allocated pulses are inadequate in their CPIs. Fig. $7 d-f$ shows the reconstructed images when the allocating proportions of pulses are calculated according to both sizes and rotation velocities of three targets. Images with better quality are obtained for all targets.

The results of the simulations and comparison are consistent with the theoretical analysis in this paper. Hence, the proposed multi-target imaging method is proved to be reasonable and effective.

\section{Conclusions}

In order to implement multi-target simultaneous ISAR imaging, an observing strategy and CS imaging method is proposed in this paper. The CS theory is introduced into multi-target imaging, and a random pulse allocating method is proposed based on the flexibility of PAR. Multi-target simultaneous imaging is realized by controlling the beam directions of radar pulses, without changing the architecture and working mode of PARs. The method can take full advantage of radar resources and obtain images for multiple targets using much less pulses than conventional RD method. Although every target gets very limited pulses, the sparsity of ISAR images and the theory of CS guarantee effective reconstruction of images. The allocation proportion is calculated based on the sizes and rotation velocities of targets. A self-adapting observing flow is designed for practical radar applications, which improves the robustness of the proposed method. SL0 algorithm is employed to reconstruct images of targets from random pulses. The simulation results testify the conclusion in this paper and prove the feasibility of the proposed method.

\section{Competing interests}

The authors declare that they have no competing interests.

\section{Acknowledgements}

This work was supported by the National Natural Science Foundation of China (Grant Nos. 61471373).

Received: 24 September 2015 Accepted: 18 February 2016 Published online: 24 February 2016

1. C Ozdemir, Inverse Synthetic Aperture Radar Imaging With MATLAB Algorithms (John Wileyand Sons, INC, Hoboken, New Jersey, 2012)

2. F Zhou, X Bai, M Xing, Z Bao, Analysis of wide-angle radar imaging. IET Radar, Sonar and Navigation 5(4), 449-457 (2011)

3. Pirkl M. and Holpp W., From research to application: how phased array radars conquered the real world, 14th International Radar Symposium, pp.17-22, 2013

4. M Zatman., in Proceedings of IEEE Radar Conference. Radar resource management for UESA( IEEE Radar Conference, Long Beach, California, 2002) 5. DL Donoho, Compressed sensing. IEEE Trans Inf Theory 52(4), 1289-1306 (2006)

6. EJ Cand'es, J Romberg, T Tao, Robust uncertainty principles: exact signal reconstruction from highly incomplete frequency information. IEEE Trans Inf Theory 52, 489-509 (2006)
} 
7. MA Herman, T Strohmer, High-resolution radar via compressed sensing. IEEE Trans Signal Process 57, 2275-2284 (2009)

8. JHG Ender, On compressive sensing applied to radar. Signal Process 90, 1402-1414 (2010)

9. X Bai, F Zhou, M Xing, Z Bao, High-resolution radar imaging of air-targets from sparse azimuth data. IEEE Trans on Aerospace and Electronic Systems 48(2), 1643-1655 (2012)

10. MG Amin, F Ahmad, Compressive sensing for through-the-wall radar imaging. J Electronic Imaging 22, 030901 (2013)

11. D Li, X Li, Y Cheng, Y Qin, H Wang, Radar coincidence imaging in the presence of target-motion-induced error. J Electronic Imaging 23, 023014 (2014)

12. L Zhang, M Xing, C-W Qiu, J Li, Z Bao, Achieving higher resolution ISAR imaging with limited pulses via compressed sampling. IEEE Geosci Remote Sens Lett 6, 567-571 (2009)

13. JA Tropp, AC Gilbert, Signal recovery from random measurements via orthogonal matching pursuit. IEEE Trans Inf Theory 53, 4655-4666 (2007)

14. EJ Candes, The restricted isometry property and its implications for compressed sensing. C R Acad Sci Paris, Ser I 346, 589-592 (2008)

15. J Wang, D Kasilingam, Global range alignment for ISAR. IEEE Trans Aerosp Electron Syst 39, 351-357 (2003)

16. $Y$ Wang, $H$ Ling, $V$ Chen, ISAR motion compensation via adaptive joint time-frequency technique. IEEE Trans Aerosp Electron Syst 34, 670-677 (1998)

17. RP Perry, RC Dipietro, R Fante, in Proceedings of IEEE National Radar Conference. Coherent Integration With Range Migration Using Keystone Formatting(IEEE National Radar Conference, Boston, MA,2007)

18. M Xing, R Wu, J Lan, Z Bao, Migration through resolution cell compensation in ISAR imaging. IEEE Geosci Remote Sens Lett 1, 141-144 (2004)

19. J Ender, in Proceedings of 14th International IEEE Radar Symposium. A brief review of compressive sensing applied to radar. (IRS 2013, Dresden, Germany, 2013)

20. R Lambour, N Rajan, T Morgan, I Kupiec, E Stansbery, Assessment of orbital debris size estimation from radar cross-section measurements. Adv Space Res 34(No. 5), 1013-1020 (2004). Space Debris

21. $Y$ Wang, $Y$ Jiang, A novel algorithm for estimating the rotation angle in isar imaging. IEEE Geosci Remote Sens Lett 5, 608-609 (2008)

22. D Zhu, X Yu, Z Zhu, in Proceedings of 2011 IEEE CIE International Conference on Radar. Algorithms for compressed ISAR autofocusing. (2011 IEEE CIE International Conference on Radar,Chengdu,China, 2011)

23. D Zhu, L Wang, Y Yu, Q Tao, Z Zhu, Robust isar range alignment via minimizing the entropy of the average range profile. IEEE Geosci Remote Sens Lett 6, 204-208 (2009)

24. Q Hou, L Fan, S Su, Z Chen, Compensation of phase errors for compressed sensing based ISAR imagery using inadequate pulses. Progress In Electromagnetics Research M 41, 125-138 (2015)

25. S Ji, Y Xue, L Carin, Bayesian compressive sensing. IEEE Transctions on Signal Processing 56, 2346-2356 (2008)

26. GH Mohimani, M Babaie-Zadeh, C Jutten, A fast approach for overcomplete sparse decomposition based on smoothed 10 norm. IEEE Trans Signal Process 57, 289-301 (2007)

27. J Liu, $S X u, X$ Gao, X Li, Compressive radar imaging methods based on fast smoothed 10 algorithm. 2012 International Workshop on Information and Electronics Engineering 29, 2209-2213 (2012). Elsevier Ltd

\section{Submit your manuscript to a SpringerOpen ${ }^{\mathcal{O}}$ journal and benefit from:}

- Convenient online submission

Rigorous peer review

- Immediate publication on acceptance

- Open access: articles freely available online

- High visibility within the field

- Retaining the copyright to your article

Submit your next manuscript at $\boldsymbol{s p r i n g e r o p e n . c o m ~}$ 